\title{
The Boundedness of the Hardy-Littlewood Maximal Operator and Multilinear Maximal Operator in Weighted Morrey Type Spaces
}

\author{
Takeshi Iida \\ Department of General Education, Fukushima National College of Technology, Fukushima 970-8034, Japan \\ Correspondence should be addressed to Takeshi Iida; tiida@fukushima-nct.ac.jp
}

Received 25 November 2013; Accepted 9 January 2014; Published 16 March 2014

Academic Editor: Vagif Guliyev

Copyright (C) 2014 Takeshi Iida. This is an open access article distributed under the Creative Commons Attribution License, which permits unrestricted use, distribution, and reproduction in any medium, provided the original work is properly cited.

The aim of this paper is to prove the boundedness of the Hardy-Littlewood maximal operator on weighted Morrey spaces and multilinear maximal operator on multiple weighted Morrey spaces. In particular, the result includes the Komori-Shirai theorem and the Iida-Sato-Sawano-Tanaka theorem for the Hardy-Littlewood maximal operator and multilinear maximal function.

\section{Introduction}

The aim of this paper is to investigate the boundedness of the Hardy-Littlewood maximal operator on weighted Morrey spaces and multilinear maximal operator on weighted Morrey type spaces. The Hardy-Littlewood maximal function $M f$ is given by

$$
M f(x):=\sup _{\substack{Q \ni x \\ Q: \text { cube }}} f_{Q}|f(y)| d y,
$$

where $x \in \mathbb{R}^{n}, f$ is locally integrable function, and the description $f_{Q}$ is an integral mean on $Q$ :

$$
f_{\mathrm{Q}} F(y) d y:=\frac{1}{|\mathrm{Q}|} \int_{\mathrm{Q}} F(y) d y \text {. }
$$

In this paper, for every cube $Q \subset \mathbb{R}^{n}$, we assume that the sides of $Q$ are parallel to the coordinate axes and $|Q|$ describes the volume of $Q$. A weight is a nonnegative locally integrable function on $\mathbb{R}^{n}$ that takes values in $(0, \infty)$ almost everywhere. Given a weight $w$ and a measurable set $E$, we use the notation

$$
w(E):=\int_{E} w(x) d x
$$

to denote the $w$-measure of the set $E$. We recall weighted Lebesgue spaces, Morrey spaces, weighted Morrey spaces, and multi-Morrey spaces.
Definition 1 (weighted Lebesgue spaces). Let $0<p<\infty$ and let $w$ be a weight. The weighted Lebesgue space is defined by the norm (or quasinorm)

$$
\|f\|_{L^{p}(w)}:=\left(\int_{\mathbb{R}^{n}}|f(x)|^{p} w(x) d x\right)^{1 / p} .
$$

Definition 2 (Morrey spaces). For $0<p \leq p_{0}<\infty$, the Morrey space $\mathscr{M}_{p}^{p_{0}}\left(\mathbb{R}^{n}\right)$ is defined by the norm (or quasinorm)

$$
\|f\|_{\mathscr{M}_{p}^{\mathrm{p}_{0}}\left(\mathbb{R}^{n}\right)}=\sup _{\substack{\mathrm{Q} \in \mathbb{R}^{n} ; \\ \text { Q:cube }}}|Q|^{1 / p_{0}}\left(f_{\mathrm{Q}}|f(x)|^{p} d x\right)^{1 / p} .
$$

Definition 3 (weighted Morrey spaces). Let $w_{1}$ and $w_{2}$ be weights. For $0<p \leq p_{0}<\infty$, the weighted Morrey space is defined by the norm (or quasinorm)

$$
\begin{aligned}
& \|f\|_{\mathscr{M}_{p}^{p_{0}}\left(w_{1}, w_{2}\right)} \\
& \quad:=\sup _{\substack{\mathrm{Q} \subset \mathbb{R}^{n} ; \\
Q: c u b e}} w_{1}(Q)^{1 / p_{0}}\left(\frac{1}{w_{1}(Q)} \int_{Q}|f(x)|^{p} w_{2}(x) d x\right)^{1 / p} .
\end{aligned}
$$

Definition 4 (multi-Morrey space). Let $0<p_{1}, \ldots, p_{m}<\infty$ and $1 / p_{0} \leq 1 / p_{1}+\cdots+1 / p_{m}$. The multi-Morrey space 
is defined by the quantity for vector valued function $\vec{f}=$ $\left(f_{1}, \ldots, f_{m}\right)$ :

$$
\|\vec{f}\|_{\mathscr{M}_{\vec{P}}^{p_{0}}\left(\mathbb{R}^{n}\right)}:=\sup _{\substack{Q \subset \mathbb{R}^{n} ; \\ Q: c u b e}}|Q|^{1 / p_{0}} \prod_{j=1}^{m}\left(f_{Q}\left|f_{j}\left(y_{j}\right)\right|^{p_{j}} d y_{j}\right)^{1 / p_{j}} .
$$

Hence it is natural to consider multiple weighted Morrey spaces.

Definition 5 (multiple weighted Morrey spaces). Let $0<p_{1}$, $\ldots, p_{m}<\infty$ and $1 / p_{0} \leq 1 / p_{1}+\cdots+1 / p_{m}$. Let $\vec{w}=\left(w_{1}\right.$, $\ldots, w_{m}$ ) be a multiple weight. Let $W$ be a weight. The multiple weighted Morrey space is defined by the quantity for vector valued function $\vec{f}=\left(f_{1}, \ldots, f_{m}\right)$ :

$$
\begin{aligned}
& \|\vec{f}\|_{\mathscr{M}_{\vec{p}}^{p_{0}}(W, \vec{w})} \\
& \quad:=\sup _{\substack{\mathrm{Q} \subset \mathbb{R}^{n} ; \\
\text { Q:cube }}} W(Q)^{1 / p_{0}} \prod_{j=1}^{m}\left(\frac{1}{W(Q)} \int_{Q}\left|f_{j}\left(y_{j}\right)\right|^{p_{j}} w_{j}\left(y_{j}\right) d y_{j}\right)^{1 / p_{j}} .
\end{aligned}
$$

In this framework, we investigate the well-known results. The following theorem which is elementary result was discovered by Muckenhoupt [1].

Theorem A. Let $w$ be a weight and $1<p<\infty$ :

$$
\|M f\|_{L^{p}(w)} \leq C\|f\|_{L^{p}(w)}
$$

if and only if

$$
w \in A_{p}\left(\mathbb{R}^{n}\right),
$$

where

$$
[w]_{A_{p}}:=\sup _{\substack{\mathrm{Q} \subset \mathbb{R}^{n} ; \\ \mathrm{Q}: c u b e}}\left(f_{\mathrm{Q}} w(x) d x\right)\left(f_{\mathrm{Q}} w(x)^{1 /(1-p)} d x\right)^{p-1}<\infty .
$$

Komori and Shirai [2] introduced the weighted Morrey spaces and proved the following theorem. The following theorem gives us the boundedness of the Hardy-Littlewood maximal operator on weighted Morrey spaces.

Theorem B. Let $w$ be a weight and $1<p<p_{0}<\infty$. If $w \in A_{p}\left(\mathbb{R}^{n}\right)$, then one has

$$
\|M f\|_{\mathscr{M}_{p}^{p_{0}}(w, w)} \leq C\|f\|_{\mathscr{M}_{p}^{p_{0}}(w, w)} .
$$

On the other hand, in [3], the following theorem was proved. The following result gives also the boundedness of the Hardy-Littlewood maximal operator on weighted Morrey spaces.
Theorem C. Let $w$ be a weight and $1<p \leq p_{0}<\infty$. If

$$
\begin{aligned}
& \sup _{\substack{Q<Q^{\prime} ; \\
Q, Q^{\prime}: \text { cubes }}}\left(\frac{|Q|}{\left|Q^{\prime}\right|}\right)^{1 / p_{0}}\left(f_{Q} w(x)^{p} d x\right)^{1 / p}\left(f_{Q^{\prime}} w(x)^{-p^{\prime}} d x\right)^{1 / p^{\prime}} \\
& \quad<\infty,
\end{aligned}
$$

then one has

$$
\|M f\|_{\mathscr{U}_{p}^{p_{0}}\left(1, w^{p}\right)} \leq C\|f\|_{\mathscr{M}_{p}^{p_{0}}\left(1, w^{p}\right)^{p}}
$$

Moreover we can extend Theorem $\mathrm{C}$ to the multilinear version (see $[3,4])$.

Theorem D. Let $1<p_{1}, \ldots, p_{m}<\infty$ and $1 / p_{0} \leq 1 / p_{1}+\cdots+$ $1 / p_{m}$. Let $\vec{w}=\left(w_{1}, \ldots, w_{m}\right)$ be a multiple weight. If

$$
\begin{gathered}
\sup _{\substack{Q \subset Q^{\prime} ; \\
Q, Q^{\prime}: c u b e s}}\left(\frac{|Q|}{\left|Q^{\prime}\right|}\right)^{1 / p_{0}}\left(f_{Q}\left(w_{1} \cdots w_{m}\right)(x)^{p} d x\right)^{1 / p} \\
\quad \times \prod_{j=1}^{m}\left(f_{Q^{\prime}} w_{j}\left(y_{j}\right)^{-p^{\prime}} d y_{j}\right)^{1 / p^{\prime}}<\infty .
\end{gathered}
$$

Then one has

$$
\|\mathscr{M} \vec{f}\|_{\mathscr{M}_{p}^{p_{0}}\left(1,\left(w_{1} \cdots w_{m}\right)^{p}\right)} \leq C\|f\|_{\mathscr{M}_{p}^{p_{0}}(1, \vec{w} \vec{p})},
$$

where $\vec{w} \vec{P}=\left(w_{1}^{p_{1}}, \ldots, w_{m}^{p_{m}}\right)$ and

$$
\mathscr{M} \vec{f}(x):=\sup _{\mathrm{Q} \ni x} \prod_{j=1}^{m} f_{\mathrm{Q}}\left|f_{j}\left(y_{j}\right)\right| d y_{j} .
$$

Because the ways of the evaluation by the weighted Morrey norm are different, Theorems $\mathrm{B}$ and $\mathrm{C}$ are independent. Therefore, it is natural to consider unifying Theorems $\mathrm{B}$ and C. The question is not settled yet. In this paper, we unify Theorems B-D.

\section{Main Results}

Theorem 6. Let $1<p \leq p_{0}<\infty$ and let $w_{1}$ and $w_{2}$ be weights. Additionally assume that $w_{1}$ satisfies the doubling condition: there exists $C>0$ such that

$$
w_{1}(2 Q) \leq C w_{1}(Q) .
$$

Assume that the following condition holds:

$$
\begin{aligned}
{\left[w_{1}, w_{2}\right]_{p_{0}, p}=} & \sup _{\substack{\mathrm{QcQ^{ \prime }} \\
Q, Q^{\prime}: \text { cubes }}}\left(\frac{w_{1}(Q)}{w_{1}\left(Q^{\prime}\right)}\right)^{1 / p_{0}-1 / p}\left(\frac{|Q|}{\left|Q^{\prime}\right|}\right)^{1 / p} \\
& \times\left(f_{Q} w_{2}(x)^{p} d x\right)^{1 / p}\left(f_{Q^{\prime}} w_{2}(x)^{-p^{\prime}} d x\right)^{1 / p^{\prime}} \\
< & \infty .
\end{aligned}
$$


Then, one has

$$
\begin{gathered}
w_{1}(Q)^{1 / p_{0}}\left(\frac{1}{w_{1}(Q)} \int_{Q} M f(x)^{p} w_{2}(x)^{p} d x\right)^{1 / p} \\
\leq C\left[w_{1}, w_{2}\right]_{p_{0}, p} \sup _{\substack{\mathrm{Q}^{\prime} Q^{\prime} \\
Q, Q^{\prime} \text { cubes }}} w_{1}\left(Q^{\prime}\right)^{1 / p_{0}} \\
\quad \times\left(\frac{1}{w_{1}\left(Q^{\prime}\right)} \int_{Q^{\prime}}|f(x)|^{p} w_{2}(x)^{p} d x\right)^{1 / p} .
\end{gathered}
$$

Remark 7. If condition (19) holds, then $w_{2}^{p} \in A_{p}\left(\mathbb{R}^{n}\right)$. In fact, for every cube $Q \subset \mathbb{R}^{n}$,

$$
\begin{aligned}
\left(f_{\mathrm{Q}} w_{2}(x)^{p} d x\right)^{1 / p}\left(f_{\mathrm{Q}} w_{2}(x)^{-p^{\prime}} d x\right)^{1 / p^{\prime}} \\
=\left(\frac{w_{1}(Q)}{w_{1}(Q)}\right)^{1 / p_{0}-1 / p}\left(\frac{|Q|}{|Q|}\right)^{1 / p}\left(f_{\mathrm{Q}} w_{2}(x)^{p} d x\right)^{1 / p} \\
\quad \times\left(f_{\mathrm{Q}} w_{2}(x)^{-p^{\prime}} d x\right)^{1 / p^{\prime}} \\
\leq\left[w_{1}, w_{2}\right]_{p_{0}, p}<\infty .
\end{aligned}
$$

Therefore, we obtain $w_{2}^{p} \in A_{p}\left(\mathbb{R}^{n}\right)$.

Theorem 8. Let $w_{1}$ and $w_{2}$ be weights. Let $1<p \leq p_{0}<\infty$. Additionally assume that $w_{1}$ satisfies the doubling condition. If $\left[w_{1}, w_{2}\right]_{p_{0}, p}<\infty$, then one has

$$
\|M f\|_{\mathscr{M}_{p}^{p_{0}}\left(w_{1}, w_{2}^{p}\right)} \leq C\left[w_{1}, w_{2}\right]_{p_{0}, p}\|f\|_{\mathscr{M}_{p}^{p_{0}}\left(w_{1}, w_{2}^{p}\right)}
$$

Remark 9. Theorem 8 includes Theorems $\mathrm{B}$ and C. In Theorem 8 , if $w_{1}=1$, then Theorem 8 corresponds with Theorem C. In Theorem 8 , if $w_{1}=w_{2}^{p}=w$, then Theorem 8 corresponds with Theorem B. In fact, condition (19) corresponds with $w \in A_{p}\left(\mathbb{R}^{n}\right)$. We state the detail:

$$
\begin{aligned}
& {\left[w, w^{1 / p}\right]_{p_{0}, p}} \\
& =\sup _{\substack{\mathrm{Q}^{\prime} Q^{\prime} \\
Q, Q^{\prime}: \text { cubes }}}\left(\frac{w(Q)}{w\left(Q^{\prime}\right)}\right)^{1 / p_{0}-1 / p}\left(\frac{|Q|}{\left|Q^{\prime}\right|}\right)^{1 / p} \\
& \quad \times\left(f_{\mathrm{Q}} w(x) d x\right)^{1 / p}\left(f_{\mathrm{Q}^{\prime}} w(x)^{-p^{\prime} / p} d x\right)^{1 / p^{\prime}}
\end{aligned}
$$

$$
\begin{aligned}
& =\sup _{\substack{\mathrm{Q}^{\prime} Q^{\prime} \\
Q, Q^{\prime}: \text { cubes }}}\left(\frac{w(Q)}{w\left(Q^{\prime}\right)}\right)^{1 / p_{0}}\left(\frac{w\left(Q^{\prime}\right)}{\left|Q^{\prime}\right|}\right)^{1 / p} \\
& \times\left(f_{Q^{\prime}} w(x)^{-p^{\prime} / p} d x\right)^{1 / p^{\prime}} \\
& =\sup _{\substack{\mathrm{Q}^{\prime} Q^{\prime} \\
\mathrm{Q}^{\prime} \mathrm{Q}^{\prime} \text { cubes }}}\left(\frac{w(Q)}{w\left(Q^{\prime}\right)}\right)^{1 / p_{0}} \\
& \times\left[\left(f_{\mathrm{Q}^{\prime}} w(x) d x\right)\left(f_{\mathrm{Q}^{\prime}} w(x)^{1 /(1-p)} d x\right)^{p-1}\right]^{1 / p} \\
& \leq[w]_{A_{p}}^{1 / p} . \sup _{\substack{\mathrm{Q}_{\mathrm{Q}} \\
\mathrm{Q} \mathrm{Q}^{\prime}: \text { cubes }}}\left(\frac{w(\mathrm{Q})}{w\left(\mathrm{Q}^{\prime}\right)}\right)^{1 / p_{0}} \\
& \leq[w]_{A_{p}}^{1 / p}<\infty .
\end{aligned}
$$

Theorem 10. Let $w_{1}, w_{2}$, and $v$ be weights. Additionally assume that $w_{1}$ satisfies the doubling condition. Let $1<p \leq$ $p_{0}<\infty, a>1$, and

$$
\begin{aligned}
{\left[w_{1}, w_{2}, v\right]_{p_{0}, p, p / a} } & :=\sup _{\substack{Q_{Q} Q^{\prime} \\
Q, Q^{\prime}: \text { cubes }}}\left(\frac{w_{1}(Q)}{w_{1}\left(Q^{\prime}\right)}\right)^{1 / p_{0}-1 / p}\left(\frac{|Q|}{\left|Q^{\prime}\right|}\right)^{1 / p} \\
& \times\left(f_{Q} v(y)^{p} d y\right)^{1 / p}\left(f_{Q^{\prime}} w_{2}(y)^{-(p / a)^{\prime}} d y\right)^{1 /(p / a)^{\prime}} \\
< & \infty .
\end{aligned}
$$

Then one has

$$
\|M f\|_{\mathscr{M}_{p}^{p_{0}}\left(w_{1}, v^{p}\right)} \leq C\left[w_{1}, w_{2}, v\right]_{p_{0}, p, p / a}\|f\|_{\mathscr{M}_{p}^{p_{0}}\left(w_{1}, w_{2}^{p}\right)} .
$$

We can extend Theorem 10 as follows.

Theorem 11. Let $w_{1}, w_{2}$, and $v$ be weights. Additionally assume that $w_{1}$ satisfies the doubling condition. Let $1<p \leq p_{0} \leq q_{0}<$ $\infty, a>1$, and

$$
\begin{aligned}
{\left[w_{1}, w_{2}, v\right]_{p_{0}, q_{0}, p, p / a} } & : \sup _{\substack{\mathrm{QcQ}^{\prime} \\
\mathrm{Q}, \mathrm{Q}^{\prime}: \text { cubes }}} \frac{w_{1}(\mathrm{Q})^{1 / q_{0}}}{w_{1}\left(\mathrm{Q}^{\prime}\right)^{1 / p_{0}}}\left(\frac{w_{1}\left(Q^{\prime}\right)}{w_{1}(\mathrm{Q})}\right)^{1 / p}\left(\frac{|Q|}{\left|Q^{\prime}\right|}\right)^{1 / p} \\
& \times\left(f_{\mathrm{Q}} v(y)^{p} d y\right)^{1 / p}\left(f_{\mathrm{Q}^{\prime}} w_{2}(y)^{-(p / a)^{\prime}} d y\right)^{1 /(p / a)^{\prime}} .
\end{aligned}
$$$$
<\infty \text {. }
$$ 
Then one has

$$
\|M f\|_{\mathscr{M}_{p}^{q_{0}}\left(w_{1}, v^{p}\right)} \leq C\left[w_{1}, w_{2}, v\right]_{p_{0}, q_{0}, p, p / a}\|f\|_{\mathscr{M}_{p}^{p_{0}}\left(w_{1}, w_{2}^{p}\right)} .
$$

By considering the multilinear version, we obtain the following theorems.

Theorem 12. Let $1<p_{1}, \ldots, p_{m}<\infty, 1 / p=1 / p_{1}+\cdots+$ $1 / p_{m}$, and $a>1$. Let $v$ and $W$ be weights. Let $\vec{w}=\left(w_{1}, \ldots, w_{m}\right)$ be a multiple weight. Additionally assume that $W$ satisfies the doubling condition and

$$
\begin{aligned}
{[v, W, \vec{w}]_{p_{0}, p, \vec{P} / a}:=} & \sup _{\substack{Q \subset Q^{\prime} \\
Q, Q^{\prime}: c u b e s}}\left(\frac{W(Q)}{W\left(Q^{\prime}\right)}\right)^{1 / p_{0}-1 / p}\left(\frac{|Q|}{\left|Q^{\prime}\right|}\right)^{1 / p} \\
& \times\left(f_{Q} v(x)^{p} d x\right)^{1 / p} \\
& \times \prod_{j=1}^{m}\left(f_{Q^{\prime}} w_{j}\left(y_{j}\right)^{-\left(p_{j} / a\right)^{\prime}} d y_{j}\right)^{1 /\left(p_{j} / a\right)^{\prime}} \\
< & \infty .
\end{aligned}
$$

Then one has

$$
\|\mathscr{M} \vec{f}\|_{\mathscr{M}_{p}^{p_{0}}\left(W, v^{p}\right)} \leq C\|\vec{f}\|_{\mathscr{M}_{\vec{P}}^{p_{0}}\left(W, \vec{w}^{\vec{P}}\right)},
$$

where $\vec{w}^{\vec{P}}=\left(w_{1}^{p_{1}}, \ldots, w_{m}^{p_{m}}\right)$.

We can extend Theorem 12 as follows.

Theorem 13. Let $1<p_{1}, \ldots, p_{m}<\infty, 1 / p=1 / p_{1}+\cdots+$ $1 / p_{m}, 0<p \leq p_{0} \leq q_{0}<\infty$, and $a>1$. Let $v$ and $W$ be weights. Let $\vec{w}=\left(w_{1}, \ldots, w_{m}\right)$ be a multiple weight. Additionally assume that $W$ satisfies the doubling condition and

$$
\begin{aligned}
{[v, W, \vec{w}]_{p_{0}, q_{0}, p, \vec{P} / a}:=} & \sup _{\substack{Q \subset Q^{\prime} \\
Q, Q^{\prime}: \text { cubes }}} \frac{W(Q)^{1 / q_{0}}}{W\left(Q^{\prime}\right)^{1 / p_{0}}}\left(\frac{W\left(Q^{\prime}\right)}{W(Q)}\right)^{1 / p} \\
& \times\left(\frac{|Q|}{\left|Q^{\prime}\right|}\right)^{1 / p}\left(f_{Q} v(x)^{p} d x\right)^{1 / p} \\
& \times \prod_{j=1}^{m}\left(f_{Q^{\prime}} w_{j}\left(y_{j}\right)^{-\left(p_{j} / a\right)^{\prime}} d y_{j}\right)^{1 /\left(p_{j} / a\right)^{\prime}} \\
< & <.
\end{aligned}
$$

Then one has

$$
\|\mathscr{M} \vec{f}\|_{\mathscr{M}_{p}^{q_{0}}\left(W, v^{p}\right)} \leq C\|\vec{f}\|_{\mathscr{M}_{\vec{P}}^{p_{0}}\left(W, \vec{w}^{\vec{P}}\right)^{\prime}}
$$

where $\vec{w}^{\vec{P}}=\left(w_{1}^{p_{1}}, \ldots, w_{m}^{p_{m}}\right)$

\section{Some Lemmas}

We use the following lemma (see $[3,5-9])$.

Lemma 14. Let $0<p<\infty$. Let $v$ be a weight. For every cube $Q_{0} \subset \mathbb{R}^{n}$, one has

$$
\left\|M\left(f \chi_{3 Q_{0}}\right) v\right\|_{L^{p}\left(Q_{0}\right)} \leq C\left\|\widetilde{M}_{p}^{Q_{0}}(f, v)\right\|_{L^{p}\left(Q_{0}\right)},
$$

where

$$
\begin{aligned}
& \widetilde{M}_{p}^{Q_{0}}(f, v)(x) \\
& \quad:=\sup _{Q_{0} \supset Q_{\ni}}\left(f_{3 Q}|f(y)| d y\right)\left(f_{Q} v(y)^{p} d y\right)^{1 / p} .
\end{aligned}
$$

Moreover we can extend Lemma 14 to the multilinear maximal function.

Lemma 15. Let $0<p<\infty$. Let $v$ be a weight. For every cube $Q_{0} \subset \mathbb{R}^{n}$, one has

$$
\left\|\mathscr{M}\left(f_{1} \chi_{3 Q_{0}}, \ldots, f_{m} \chi_{3 Q_{0}}\right) v\right\|_{L^{p}\left(Q_{0}\right)} \leq C\left\|\widetilde{M}_{p}^{\mathrm{Q}_{0}}(\vec{f}, v)\right\|_{L^{p}\left(\mathrm{Q}_{0}\right)},
$$

where

$$
\begin{aligned}
& \widetilde{M}_{p}^{Q_{0}}(\vec{f}, v)(x) \\
& \left.:=\sup _{Q_{0} \supset Q_{\supset} \prod_{j=1}^{m}} \prod_{j=1}\left|f_{j}\left(y_{j}\right)\right| d y_{j}\right)\left(f_{Q} v(y)^{p} d y\right)^{1 / p} .
\end{aligned}
$$

Remark 16. In [3], there is not the restriction $Q \subset Q_{0}$ in the maximal function $\widetilde{M}_{p}^{\mathrm{Q}_{0}}(\vec{f}, v)$. However, reexamining the argument of the proof in [3], we can remove the restriction. Hence the norm inequalities in Lemmas 14 and 15 are sharper than the norm inequalities in [3].

\section{Proof of Theorems}

4.1. Onelinear Version. We prove Theorem 11. We omit the proof of Theorems 6, 8, and 10 .

Proof of Theorem 11. For every cube $Q_{0} \subset \mathbb{R}^{n}$, by sublinearity, $M f(x) \leq M\left(f \chi_{3 Q_{0}}\right)(x)+M\left(f \chi_{\left(3 Q_{0}\right)^{c}}\right)(x)$. Let $f_{0}=f \chi_{3 Q_{0}}$ and $f_{\infty}=f \chi_{\left(3 Q_{0}\right)^{c}}$. By Lemma 14,

$$
\left\|\left(M f_{0}\right) v\right\|_{L^{p}\left(\mathrm{Q}_{0}\right)} \leq C\left\|\widetilde{M}_{p}^{\mathrm{Q}_{0}}(f, v)\right\|_{L^{p}\left(\mathrm{Q}_{0}\right)} .
$$


By Hölder's inequality, we have

$$
\begin{aligned}
\widetilde{M}_{p}^{Q_{0}}(f, v)(x) & \\
\leq & \sup _{x \in Q_{Q} Q_{0}}\left(f_{3 Q}\left|f(y) w_{2}(y)\right|^{p / a} d y\right)^{a / p} \\
& \times\left(f_{3 Q} w_{2}(y)^{-(p / a)^{\prime}} d y\right)^{1 /(p / a)^{\prime}}\left(f_{Q} v(y)^{p} d y\right)^{1 / p} \\
= & \sup _{x \in Q_{0}} \frac{w_{1}(Q)^{1 / q_{0}}}{w_{1}(3 Q)^{1 / p_{0}}}\left(\frac{w_{1}(3 Q)}{w_{1}(Q)}\right)^{1 / p}\left(\frac{|Q|}{|3 Q|}\right)^{1 / p} \\
& \times\left(f_{Q} v(y)^{p} d y\right)^{1 / p}\left(f_{3 Q} w_{2}(y)^{-(p / a)^{\prime}} d y\right)^{1 /(p / a)^{\prime}} \\
& \times \frac{w_{1}(Q)^{-1 / q_{0}}}{w_{1}(3 Q)^{-1 / p_{0}}}\left(\frac{w_{1}(3 Q)}{w_{1}(Q)}\right)^{-1 / p}\left(\frac{|3 Q|}{|Q|}\right)^{1 / p} \\
& \times\left(f_{3 Q}\left|f(y) w_{2}(y)\right|^{p / a} d y\right)^{a / p} \cdot
\end{aligned}
$$

By condition (26), we have

$$
\begin{aligned}
& \widetilde{M}_{p}^{\mathrm{Q}_{0}}(f, v)(x) \\
& \leq C\left[w_{1}, w_{2}, v\right]_{p_{0}, q_{0}, p, p / a} \sup _{x \in Q_{Q} Q_{0}} \frac{w_{1}(Q)^{-1 / q_{0}}}{w_{1}(3 Q)^{-1 / p_{0}}} \\
& \quad \times\left(\frac{w_{1}(3 Q)}{w_{1}(Q)}\right)^{-1 / p}\left(f_{3 Q}\left|f(y) w_{2}(y)\right|^{p / a} d y\right)^{a / p} .
\end{aligned}
$$

Since $w_{1}(Q) \leq w_{1}(3 Q)$, we have

$$
\begin{aligned}
\sup _{x \in Q \subset Q_{0}} & \frac{w_{1}(Q)^{-1 / q_{0}}}{w_{1}(3 Q)^{-1 / p_{0}}}\left(\frac{w_{1}(3 Q)}{w_{1}(Q)}\right)^{-1 / p} \\
& \times\left(f_{3 Q}\left|f(y) w_{2}(y)\right|^{p / a} d y\right)^{a / p} \\
= & \sup _{x \in Q \subset Q_{0}} \frac{w_{1}(3 Q)^{1 / p_{0}}}{w_{1}(Q)^{1 / q_{0}}}\left(\frac{w_{1}(Q)}{w_{1}(3 Q)}\right)^{1 / p} \\
& \times\left(f_{3 Q}\left|f(y) w_{2}(y)\right|^{p / a} d y\right)^{a / p} \\
\leq & \sup _{x \in Q \subset Q_{0}} \frac{w_{1}(3 Q)^{1 / p_{0}}}{w_{1}(Q)^{1 / q_{0}}}\left(f_{3 Q}\left|f(y) w_{2}(y)\right|^{p / a} d y\right)^{a / p} .
\end{aligned}
$$

Since $w_{1}$ satisfies the doubling condition, we obtain the following inequality; for every cube $Q \subset \mathbb{R}^{n}$,

$$
\frac{w_{1}(3 Q)^{1 / p_{0}}}{w_{1}(Q)^{1 / q_{0}}} \leq C^{1 / q_{0}} \frac{w_{1}(3 Q)^{1 / p_{0}}}{w_{1}(3 Q)^{1 / q_{0}}}=C^{1 / q_{0}} w_{1}(3 Q)^{1 / p_{0}-1 / q_{0}}
$$

Since $p_{0} \leq q_{0}$, for pair of cubes $Q \subset Q_{0}$, we have

$$
w_{1}(3 Q)^{\left(1 / p_{0}\right)-\left(1 / q_{0}\right)} \leq w_{1}\left(3 Q_{0}\right)^{\left(1 / p_{0}\right)-\left(1 / q_{0}\right)} .
$$

Hence we have

$$
\begin{aligned}
& \sup _{x \in \mathrm{QCQ}_{0}} \frac{w_{1}(Q)^{-1 / q_{0}}}{w_{1}(3 Q)^{-1 / p_{0}}}\left(\frac{w_{1}(3 Q)}{w_{1}(Q)}\right)^{-1 / p} \\
& \quad \times\left(f_{3 Q}\left|f(y) w_{2}(y)\right|^{p / a} d y\right)^{a / p} \\
& \leq C^{1 / q_{0}} w_{1}\left(3 Q_{0}\right)^{1 / p_{0}-1 / q_{0}} M\left[\left|f w_{2}\right|^{p / a} \chi_{3 Q_{0}}\right](x)^{a / p} .
\end{aligned}
$$

This implies that

$$
\begin{aligned}
&\left(\int_{Q_{0}} M f_{0}(x)^{p} v(x)^{p} d x\right)^{1 / p} \\
& \leq C\left[w_{1}, w_{2}, v\right]_{p_{0}, q_{0}, p, p / a} \cdot w_{1}\left(3 Q_{0}\right)^{1 / p_{0}-1 / q_{0}} \\
& \times\left(\int_{Q_{0}} M\left[\left|f w_{2}\right|^{p / a} \cdot \chi_{3 Q_{0}}\right](x)^{(a / p) \cdot p} d x\right)^{1 / p} \\
&= C\left[w_{1}, w_{2}, v\right]_{p_{0}, q_{0}, p, p / a} \cdot w_{1}\left(3 Q_{0}\right)^{1 / p_{0}-1 / q_{0}} \\
& \times\left(\int_{Q_{0}} M\left[\left|f w_{2}\right|^{p / a} \chi_{3 Q_{0}}\right](x)^{a} d x\right)^{1 / p} .
\end{aligned}
$$

By the boundedness of $M: L^{a}\left(\mathbb{R}^{n}\right) \rightarrow L^{a}\left(\mathbb{R}^{n}\right)$, we obtain

$$
\begin{gathered}
\left(\int_{Q_{0}} M\left[\left|f w_{2}\right|^{p / a} \chi_{3 Q_{0}}\right](x)^{a} d x\right)^{1 / p} \\
\quad \leq C\left(\int_{3 Q_{0}}\left|f(x) w_{2}(x)\right|^{p} d x\right)^{1 / p} .
\end{gathered}
$$

Since $p \leq q_{0}$, by the doubling condition of $w_{1}$, we have

$$
\begin{aligned}
& w_{1}\left(Q_{0}\right)^{1 / q_{0}-1 / p}\left(\int_{Q_{0}} M f_{0}(x)^{p} v(x)^{p} d x\right)^{1 / p} \\
& \leq C\left[w_{1}, w_{2}, v\right]_{p_{0}, q_{0}, p, p / a} \\
& \cdot w_{1}\left(Q_{0}\right)^{1 / q_{0}-1 / p} w_{1}\left(3 Q_{0}\right)^{1 / p_{0}-1 / q_{0}} \\
& \times\left(\int_{3 Q_{0}}\left|f(x) w_{2}(x)\right|^{p} d x\right)^{1 / p} \\
&\left.\leq C\left[w_{1}, w_{2}, v\right]_{p_{0}, q_{0}, p, p / a}\left|f(x) w_{2}(x)\right|^{p} d x\right)^{1 / p} \\
& \cdot w_{1}\left(3 Q_{0}\right)^{1 / p_{0}-1 / p}\left(\left.\int_{3 Q_{0}}\left|f\left(w_{1}, w_{2}, v\right]_{p_{0}, q_{0}, p, p / a}\right| f(x) w_{2}(x)\right|^{p} d x\right)^{1 / p} . \\
& \leq C\left[w _ { 1 } ( 3 Q _ { 0 } ) ^ { 1 / p _ { 0 } - 1 / p } \left(\int_{3 Q_{0}} \mid\right.\right.
\end{aligned}
$$


Taking the weighted Morrey norm, we obtain the following inequality:

$$
\left\|M f_{0}\right\|_{\mathscr{M}_{p}^{q_{0}}\left(w_{1}, v^{p}\right)} \leq C\|f\|_{\mathscr{M}_{p}^{p_{0}}\left(w_{1}, w_{2}^{p}\right)} .
$$

Next, we estimate $M f_{\infty}(x)$. By routine geometric observation, for $x \in Q_{0}$, we have

$$
M f_{\infty}(x) \leq C \sup _{\mathrm{Q} \supset \mathrm{Q}_{0}} f_{\mathrm{Q}}|f(y)| d y .
$$

For pair of cubes $Q \supset Q_{0}$, by Hölder's inequality, we obtain

$$
\begin{aligned}
M f_{\infty}(x) \leq & C \sup _{Q \supset Q_{0}}\left(f_{Q}|f(y)|^{p / a} w_{2}(y)^{p / a} d y\right)^{a / p} \\
& \times\left(f_{Q} w_{2}(y)^{-(p / a)^{\prime}} d y\right)^{1 /(p / a)^{\prime}} \\
\leq & C \sup _{Q \supset Q_{0}} w_{1}(Q)^{1 / p_{0}-1 / p} \\
& \times\left(\int_{Q}\left|f(y) w_{2}(y)\right|^{p} d y\right)^{1 / p} \frac{1}{|Q|^{1 / p}} \\
& \times\left(f_{Q} w_{2}(y)^{-(p / a)^{\prime}} d y\right)^{1 /(p / a)^{\prime}} \\
\leq & C\|f\|_{M_{p}^{p_{0}}\left(w_{1}, w_{2}^{p}\right)} \cdot \sup _{Q} \frac{1}{\left|Q Q_{0}\right|^{1 / p}} \\
& \times\left(f_{Q} w_{2}(y)^{-(p / a)^{\prime}} d y\right)^{1 /(p / a)^{\prime}} w_{1}(Q)^{1 / p-1 / p_{0}}
\end{aligned}
$$

Therefore we have

$$
\begin{aligned}
& w_{1}\left(Q_{0}\right)^{1 / q_{0}-1 / p}\left(\int_{Q_{0}} M f_{\infty}(x)^{p} v(x)^{p} d x\right)^{1 / p} \\
& \leq C\|f\|_{\mathscr{M}_{p}^{p_{0}}\left(w_{1}, w_{2}^{p}\right)} \sup _{\mathrm{Q}_{Q_{0}}} \frac{w_{1}\left(Q_{0}\right)^{1 / q_{0}}}{w_{1}(Q)^{1 / p_{0}}}\left(\frac{w_{1}(Q)}{w_{1}\left(Q_{0}\right)}\right)^{1 / p} \\
& \times\left(\frac{\left|Q_{0}\right|}{|Q|}\right)^{1 / p}\left(f_{Q_{0}} v(x)^{p} d x\right)^{1 / p} \\
& \times\left(f_{Q} w_{2}(y)^{-(p / a)^{\prime}} d y\right)^{1 /(p / a)^{\prime}}
\end{aligned}
$$

By condition (26), we have

$$
\begin{aligned}
\sup _{\substack{Q \supset Q_{0} \\
Q, Q_{0}: \text { cubes }}} & \frac{w_{1}\left(Q_{0}\right)^{1 / q_{0}}}{w_{1}(Q)^{1 / p_{0}}}\left(\frac{w_{1}(Q)}{w_{1}\left(Q_{0}\right)}\right)^{1 / p}\left(\frac{\left|Q_{0}\right|}{|Q|}\right)^{1 / p} \\
& \times\left(f_{Q_{0}} v(x)^{p} d x\right)^{1 / p}\left(f_{Q} w_{2}(y)^{-(p / a)^{\prime}} d y\right)^{1 /(p / a)^{\prime}} \\
\leq & {\left[v, w_{1}, w_{2}\right]_{p_{0}, q_{0}, p, p / a^{*}} }
\end{aligned}
$$

This implies that

$$
\begin{aligned}
& \left\|M f_{\infty}\right\|_{\mathscr{M}_{p}^{q_{0}}\left(w_{1}, v^{p}\right)} \\
& \quad \leq C\left[v, w_{1}, w_{2}\right]_{p_{0}, q_{0}, p, p / a}\|f\|_{\mathscr{M}_{p}^{p_{0}}\left(w_{1}, w_{2}^{p}\right)} .
\end{aligned}
$$

Therefore we obtain the desired result.

\subsection{Multilinear Version. We prove Theorem 13.}

Proof of Theorem 13. For every cube $Q \subset \mathbb{R}^{n}$, we have $f_{j}\left(y_{j}\right)=f_{j}\left(y_{j}\right) \chi_{3 Q_{0}}\left(y_{j}\right)+f_{j}\left(y_{j}\right) \chi_{\left(3 Q_{0}\right)^{c}}\left(y_{j}\right)=f_{j}^{0}\left(y_{j}\right)+f_{j}^{\infty}\left(y_{j}\right)$. Let $\vec{f}_{0}=\left(f_{1}^{0}, \ldots, f_{m}^{0}\right), \vec{f}_{\vec{l}}=\left(f_{1}^{l_{1}}, \ldots, f_{m}^{l_{m}}\right)$, and $\vec{l}=\left(l_{1}, \ldots, l_{m}\right)$ :

$$
\mathscr{M}(\vec{f})(x) \leq \mathscr{M}\left(\vec{f}_{0}\right)(x)+\sum_{\vec{l} \neq \overrightarrow{0}} \mathscr{M}\left(\vec{f}_{\vec{l}}\right)(x) .
$$

Firstly, we estimate $\mathscr{M}\left(\vec{f}_{0}\right)(x)$. By Lemma 15 , we have

$$
\left\|\mathscr{M}\left(\vec{f}_{0}\right) v\right\|_{L^{p}\left(Q_{0}\right)} \leq C\left\|\widetilde{M}_{p}^{Q_{0}}(\vec{f}, v)\right\|_{L^{p}\left(Q_{0}\right)},
$$

where

$$
\begin{aligned}
& \widetilde{M}_{p}^{Q_{0}}(\vec{f}, v)(x) \\
& \quad:=\sup _{Q_{0} \supset Q \ni x} \prod_{j=1}^{m}\left(f_{3 Q}\left|f_{j}\left(y_{j}\right)\right| d y_{j}\right)\left(f_{Q} v(y)^{p} d y\right)^{1 / p} .
\end{aligned}
$$

By Hölder's inequality, we obtain

$$
\begin{aligned}
\widetilde{M}_{p}^{Q_{0}}(\vec{f}, v)(x) \\
\leq \sup _{Q_{0} \supset Q_{\ni} \rightarrow x} \prod_{j=1}^{m}\left(f_{3 Q}\left|f_{j}\left(y_{j}\right)\right|^{p_{j} / a} w_{j}\left(y_{j}\right)^{p_{j} / a} d y_{j}\right)^{a / p_{j}} \\
\quad \times\left(f_{3 Q} w_{j}\left(y_{j}\right)^{-\left(p_{j} / a\right)^{\prime}} d y_{j}\right)^{1 /\left(p_{j} / a\right)^{\prime}}\left(f_{Q} v(y)^{p} d y\right)^{1 / p} .
\end{aligned}
$$

By condition (30), we obtain

$$
\begin{aligned}
& \widetilde{M}_{p}^{Q_{0}}(\vec{f}, v)(x) \\
& \leq C[v, W, \vec{w}]_{p_{0}, q_{0}, p, \vec{P} / a} \\
& \times \sup _{x \in Q_{C Q}} \prod_{j=1}^{m}\left(f_{3 Q}\left|f_{j}\left(y_{j}\right)\right|^{p_{j} / a} w_{j}\left(y_{j}\right)^{p_{j} / a} d y_{j}\right)^{a / p_{j}} \\
& \times \frac{W(3 Q)^{1 / p_{0}}}{W(Q)^{1 / q_{0}}}\left(\frac{W(Q)}{W(3 Q)}\right)^{1 / p} .
\end{aligned}
$$

Since $W(Q) \leq W(3 Q)$, we have

$$
\left(\frac{W(Q)}{W(3 Q)}\right)^{1 / p} \leq 1
$$


Since $W$ satisfies the doubling condition, we have

$$
\frac{W(3 Q)^{1 / p_{0}}}{W(Q)^{1 / q_{0}}} \leq C W(3 Q)^{1 / p_{0}-1 / q_{0}}
$$

Since $p_{0} \leq q_{0}$, for pair of cubes $Q \subset Q_{0}$, we have

$$
W(3 Q)^{1 / p_{0}-1 / q_{0}} \leq W\left(3 Q_{0}\right)^{1 / p_{0}-1 / q_{0}} .
$$

Therefore we obtain

$$
\begin{aligned}
\widetilde{M}_{p}^{\mathrm{Q}_{0}}(\vec{f}, v)(x) \\
\leq C[v, W, \vec{w}]_{p_{0}, q_{0} p, \vec{P} / a} \cdot W\left(3 Q_{0}\right)^{1 / p_{0}-1 / q_{0}} \\
\quad \cdot \sup _{x \in \mathrm{Q}_{\mathrm{Q}}} \prod_{j=1}^{m}\left(f_{3 Q}\left|f_{j}\left(y_{j}\right)\right|^{p_{j} / a} w_{j}\left(y_{j}\right)^{p_{j} / a} d y_{j}\right)^{a / p_{j}} \\
\leq C[v, W, \vec{w}]_{p_{0}, q_{0}, p, \vec{P} / a} \cdot W\left(3 Q_{0}\right)^{1 / p_{0}-1 / q_{0}} \\
\quad \cdot \prod_{j=1}^{m} M\left[\left|f_{j} w_{j}\right|^{p_{j} / a} \chi_{3 Q_{0}}\right](x)^{a / p_{j}} .
\end{aligned}
$$

This implies that

$$
\begin{aligned}
& \left(\int_{Q_{0}} \widetilde{M}_{p}^{\mathrm{Q}_{0}}(\vec{f}, v)(x)^{p} d x\right)^{1 / p} \\
& \leq C[v, W, \vec{w}]_{p_{0}, q_{0}, p, \vec{P} / a} \cdot W\left(3 Q_{0}\right)^{1 / p_{0}-1 / q_{0}} \\
& \quad \times\left(\int_{Q_{0}} \prod_{j=1}^{m} M\left[\left|f_{j} w_{j}\right|^{p_{j} / a} \chi_{3 Q_{0}}\right](x)^{\left(a / p_{j}\right) p} d x\right)^{1 / p} .
\end{aligned}
$$

Since $1 / p=1 / p_{1}+\cdots+1 / p_{m}$, by Hölder's inequality, we have

$$
\begin{aligned}
\left(\int_{\mathrm{Q}_{0}} \widetilde{M}_{p}^{\mathrm{Q}_{0}}(\vec{f}, v)(x)^{p} d x\right)^{1 / p} \\
\leq C[v, W, \vec{w}]_{p_{0}, q_{0}, p, \vec{P} / a} \cdot W\left(3 Q_{0}\right)^{1 / p_{0}-1 / q_{0}} \\
\quad \times \prod_{j=1}^{m}\left(\int_{\mathrm{Q}_{0}} M\left[\left|f_{j} w_{j}\right|^{p_{j} / a} \chi_{3 \mathrm{Q}_{0}}\right](x)^{a} d x\right)^{1 / p_{j}} .
\end{aligned}
$$

By the boundedness of $M$ on $L^{a}\left(\mathbb{R}^{n}\right) \rightarrow L^{a}\left(\mathbb{R}^{n}\right)$, we have

$$
\begin{aligned}
\left(\int_{\mathrm{Q}_{0}} \widetilde{M}_{p}^{\mathrm{Q}_{0}}(\vec{f}, v)(x)^{p} d x\right)^{1 / p} \\
\leq C[v, W, \vec{w}]_{p_{0}, q_{0}, p, \vec{P} / a} \cdot W\left(3 Q_{0}\right)^{1 / p_{0}-1 / q_{0}} \\
\quad \times \prod_{j=1}^{m}\left(\int_{3 \mathrm{Q}_{0}}\left|f_{j}(x)\right|^{p_{j}} w_{j}(x)^{p_{j}} d x\right)^{1 / p_{j}} .
\end{aligned}
$$

Since $p \leq q_{0}$, by the doubling condition of $W$, we have

$$
\begin{aligned}
W\left(Q_{0}\right)^{1 / q_{0}}\left(\frac{1}{W\left(Q_{0}\right)} \int_{Q_{0}} \mathscr{M}\left(\vec{f}_{0}\right)(x)^{p} v(x)^{p} d x\right)^{1 / p} \\
\leq C[v, W, \vec{w}]_{p_{0}, q_{0}, p, \vec{P} / a} \cdot W\left(3 Q_{0}\right)^{1 / p_{0}-1 / p} \\
\times \prod_{j=1}^{m}\left(\int_{3 Q_{0}}\left|f_{j}(x)\right|^{p_{j}} w_{j}(x)^{p_{j}} d x\right)^{1 / p_{j}} .
\end{aligned}
$$

Taking the multiple weighted Morrey quantity, we have

$$
\begin{gathered}
W\left(Q_{0}\right)^{1 / p_{0}}\left(\frac{1}{W\left(Q_{0}\right)} \int_{Q_{0}} \mathscr{M}\left(\vec{f}_{0}\right)(x)^{p} v(x)^{p} d x\right)^{1 / p} \\
\leq C[v, W, \vec{w}]_{p_{0}, q_{0}, p, \vec{P} / a} \cdot\|\vec{f}\|_{\mathscr{M}_{\vec{P}}^{p_{0}}(W, \vec{w} \vec{P})}
\end{gathered}
$$

Next, we estimate $\mathscr{M}\left(\vec{f}_{\vec{l}}\right)(x)$. By routine geometric observation, for $x \in Q_{0}$, we have

$$
\mathscr{M}\left(\vec{f}_{\vec{l}}\right)(x) \leq C \sup _{\mathrm{Q} \supset Q_{0}} \prod_{j=1}^{m} f_{Q}\left|f_{j}\left(y_{j}\right)\right| d y_{j} .
$$

By Hölder's inequality, we have

$$
\begin{aligned}
\sup _{\mathrm{Q} \supset \mathrm{Q}_{0}} \prod_{j=1}^{m} f_{\mathrm{Q}}\left|f_{j}\left(y_{j}\right)\right| d y_{j} \\
\leq \sup _{\mathrm{Q} \supset \mathrm{Q}_{0}} \prod_{j=1}^{m}\left(f_{\mathrm{Q}}\left|f_{j}\left(y_{j}\right)\right|^{p_{j} / a} w_{j}\left(y_{j}\right)^{p_{j} / a} d y_{j}\right)^{a / p_{j}} \\
\cdot\left(f_{\mathrm{Q}} w_{j}\left(y_{j}\right)^{-\left(p_{j} / a\right)^{\prime}} d y_{j}\right)^{1 /\left(p_{j} / a\right)^{\prime}} \\
\leq \sup _{\mathrm{Q} \supset \mathrm{Q}_{0}} \prod_{j=1}^{m}\left(f_{\mathrm{Q}}\left|f_{j}\left(y_{j}\right)\right|^{p_{j}} w_{j}\left(y_{j}\right)^{p_{j}} d y_{j}\right)^{1 / p_{j}} \\
\quad\left(f_{\mathrm{Q}} w_{j}\left(y_{j}\right)^{-\left(p_{j} / a\right)^{\prime}} d y_{j}\right)^{1 /\left(p_{j} / a\right)^{\prime}} .
\end{aligned}
$$

Taking the multiple weighted Morrey quantity, we obtain

$$
\begin{aligned}
\sup _{\mathrm{Q} \supset \mathrm{Q}_{0}} \prod_{j=1}^{m}\left(f_{\mathrm{Q}}\left|f_{j}\left(y_{j}\right)\right|^{p_{j}} w_{j}\left(y_{j}\right)^{p_{j}} d y_{j}\right)^{1 / p_{j}} \\
\cdot\left(f_{\mathrm{Q}} w_{j}\left(y_{j}\right)^{-\left(p_{j} / a\right)^{\prime}} d y_{j}\right)^{1 /\left(p_{j} / a\right)^{\prime}} \\
\leq C\|\vec{f}\|_{\mathscr{M}_{\vec{P}}^{p_{0}}\left(W, \vec{w}^{\vec{P}}\right)} \cdot \sup _{\mathrm{Q} \supset \mathrm{Q}_{0}} \frac{1}{|Q|^{1 / p}} W(Q)^{1 / p-1 / p_{0}} \\
\times \prod_{j=1}^{m}\left(f_{Q} w_{j}\left(y_{j}\right)^{-\left(p_{j} / a\right)^{\prime}} d y_{j}\right)^{1 /\left(p_{j} / a\right)^{\prime}} .
\end{aligned}
$$


This implies that

$$
\begin{aligned}
W\left(Q_{0}\right)^{1 / q_{0}}\left(\frac{1}{W\left(Q_{0}\right)} \int_{Q_{0}} \mathscr{M}\left(\vec{f}_{\vec{l}}\right)(x)^{p} v(x)^{p} d x\right)^{1 / p} \\
\leq C\|\vec{f}\|_{\mathscr{M}_{\vec{P}}^{p_{0}}\left(W, \vec{w}^{\vec{P}}\right)} \sup _{Q_{Q_{0}}} \frac{W\left(Q_{0}\right)^{1 / q_{0}}}{W(Q)^{1 / p_{0}}}\left(\frac{W(Q)}{W\left(Q_{0}\right)}\right)^{1 / p} \\
\times\left(\frac{\left|Q_{0}\right|}{|Q|}\right)^{1 / p}\left(f_{Q_{0}} v(x)^{p} d x\right)^{1 / p} \\
\quad \times \prod_{j=1}^{m}\left(f_{Q} w_{j}\left(y_{j}\right)^{-\left(p_{j} / a\right)^{\prime}} d y_{j}\right)^{1 /\left(p_{j} / a\right)^{\prime}} .
\end{aligned}
$$

By condition (28), we obtain

$$
\begin{aligned}
& W\left(Q_{0}\right)^{1 / p_{0}}\left(\frac{1}{W\left(Q_{0}\right)} \int_{Q_{0}} \mathscr{M}\left(\vec{f}_{\vec{l}}\right)(x)^{p} v(x)^{p} d x\right)^{1 / p} \\
& \leq C[v, W, \vec{w}]_{p_{0}, p, \vec{P} / a}\|\vec{f}\|_{\mathscr{M}_{\vec{P}}^{p_{0}}\left(W, \vec{w}^{\vec{P}}\right)^{\cdot}}
\end{aligned}
$$

Therefore we obtain the desired result.

\section{Conflict of Interests}

The author declares that there is no conflict of interests regarding the publication of this paper.

\section{References}

[1] B. Muckenhoupt, "Weighted norm inequalities for the Hardy maximal function," Transactions of the American Mathematical Society, vol. 165, pp. 207-226, 1972.

[2] Y. Komori and S. Shirai, "Weighted Morrey spaces and a singular integral operator," Mathematische Nachrichten, vol. 282, no. 2, pp. 219-231, 2009.

[3] T. Iida, E. Sato, Y. Sawano, and H. Tanaka, "Weighted norm inequalities for multilinear fractional operators on Morrey spaces," Studia Mathematica, vol. 205, no. 2, pp. 139-170, 2011.

[4] T. Iida, E. Sato, Y. Sawano, and H. Tanaka, "Sharp bounds for multilinear fractional integral operators on Morrey type spaces," Positivity, vol. 16, no. 2, pp. 339-358, 2012.

[5] Y. Sawano, S. Sugano, and H. Tanaka, "Generalized fractional integral operators and fractional maximal operators in the framework of Morrey spaces," Transactions of the American Mathematical Society, vol. 363, no. 12, pp. 6481-6503, 2011.

[6] Y. Sawano, S. Sugano, and H. Tanaka, "A note on generalized fractional integral operators on generalized Morrey spaces," Boundary Value Problems, vol. 2009, Article ID 835865, 18 pages, 2009.

[7] Y. Sawano, S. Sugano, and H. Tanaka, "Olsen's inequality and its applications to Schrödinger equations," in Harmonic Analysis and Nonlinear Partial Differential Equations, RIMS Kôkyûroku Bessatsu, B26, pp. 51-80, RIMS, 2011.

[8] Y. Sawano, S. Sugano, and H. Tanaka, "Orlicz-Morrey spaces and fractional operators," Potential Analysis, vol. 36, no. 4, pp. 517-556, 2012.

[9] H. Tanaka, "Morrey spaces and fractional operators," Journal of the Australian Mathematical Society, vol. 88, no. 2, pp. 247-259, 2010. 


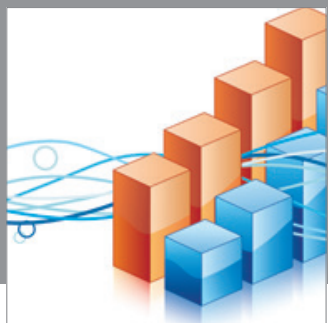

Advances in

Operations Research

mansans

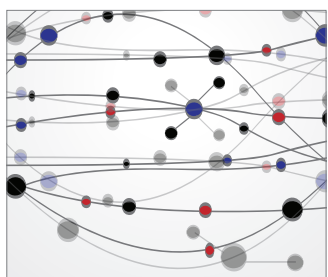

The Scientific World Journal
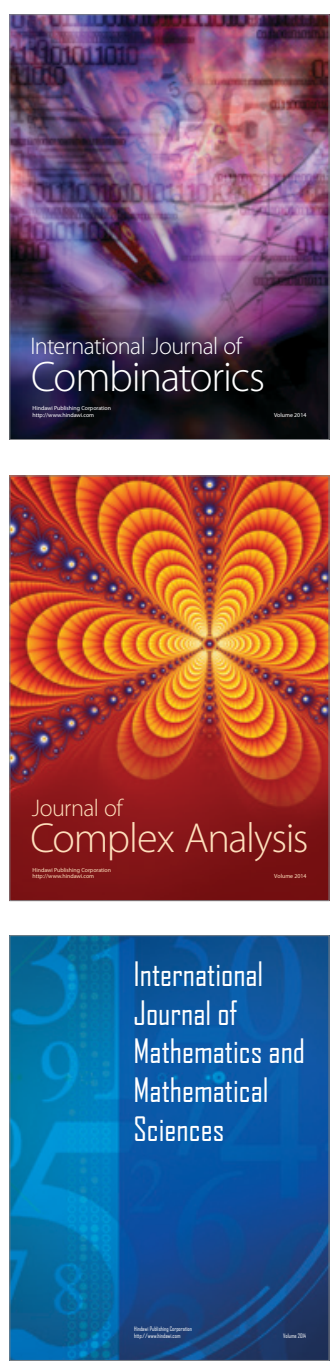
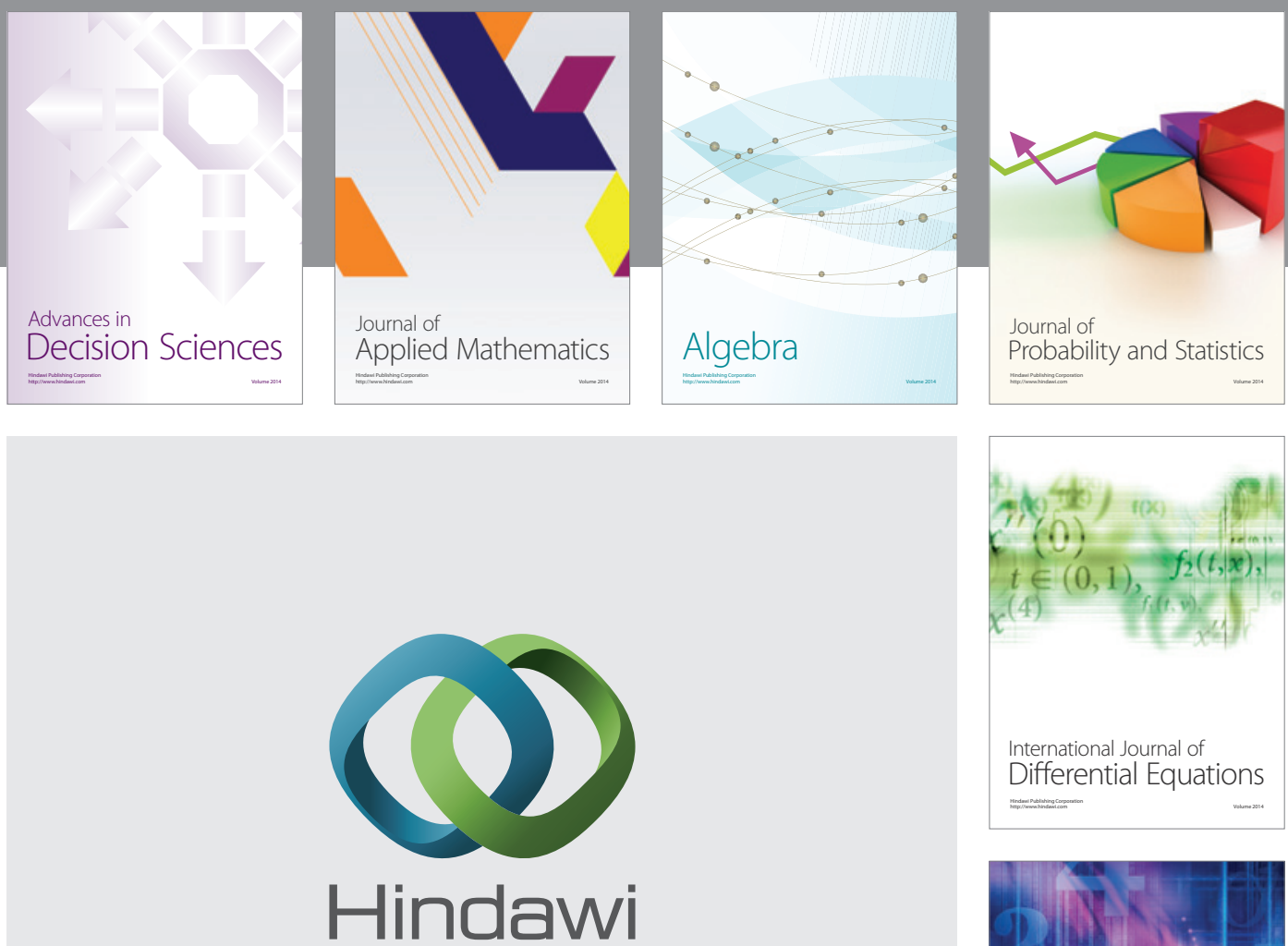

Submit your manuscripts at http://www.hindawi.com


Journal of

Function Spaces

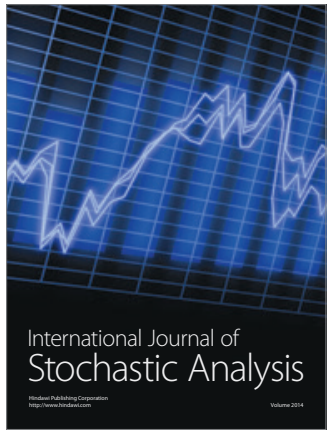

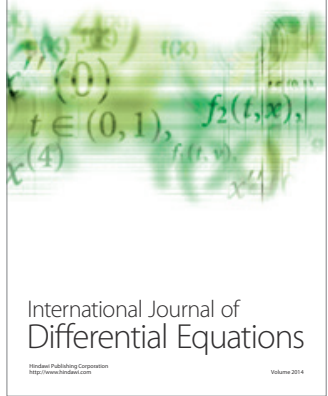
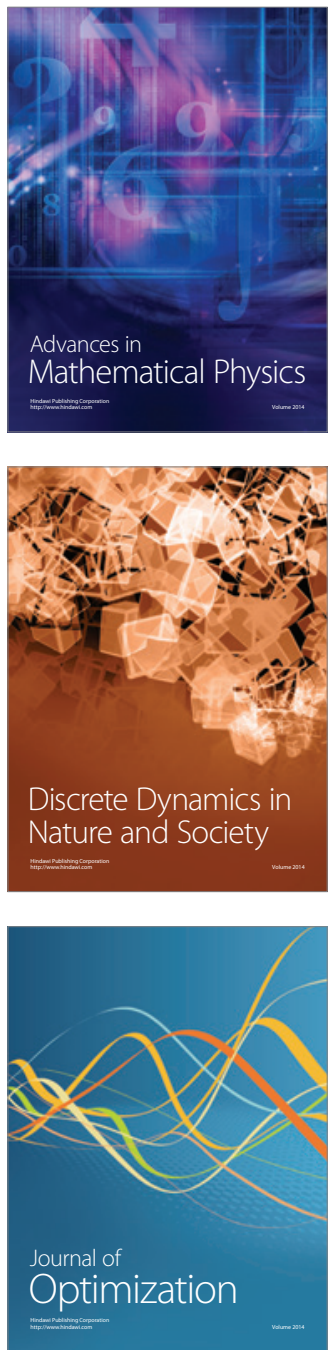